\title{
Resonance Fluorescence Spectrum of a $\Lambda$-type Quantum Emitter Close to a Metallic Nanoparticle
}

\author{
F. Carreño, ${ }^{1}$ M.A. Antón, ${ }^{1}$ V. Yannopapas, ${ }^{2}$ and E. Paspalakis ${ }^{3}$ \\ ${ }^{1}$ Facultad de Óptica y Optometría, Universidad Complutense de Madrid, \\ C/ Arcos de Jalón 118, 28037 Madrid, Spain \\ ${ }^{2}$ Department of Physics, National Technical University of Athens, Athens 157 80, Greece \\ ${ }^{3}$ Materials Science Department, School of Natural Sciences, \\ University of Patras, Patras 265 04, Greece
}

(Dated: July 6, 2016)

\begin{abstract}
We theoretically study the resonance fluorescence spectrum of a three-level quantum emitter coupled to a spherical metallic nanoparticle. We consider the case that the quantum emitter is driven by a single laser field along one of the optical transitions. We show that the development of the spectrum depends on the relative orientation of the dipole moments of the optical transitions in relation to the metal nanoparticle. In addition, we demonstrate that the location and width of the peaks in the spectrum are strongly modified by the exciton-plasmon coupling and the laser detuning, allowing to achieve controlled strongly subnatural spectral line. A strong antibunching of the fluorescent photons along the undriven transition is also obtained. Our results may be used for creating a tunable source of photons which could be used for a probabilistic entanglement scheme in the field of quantum information processing.
\end{abstract}




\section{INTRODUCTION}

The optical properties of quantum emitters have been shown to be dramatically modified when they are located in close proximity to a metallic nanoparticle (MNP). The ultracompact optical mode volume achieved in plasmon nanostructures leads to a large resonant enhancement of the local field near the MNP [1 4] , as well as the modification of spontaneous emission rates of the emitter's optical transitions [5-11]. The exciton-plasmon coupling has received a great deal of attention leading to interesting phenomena like changes in photoluminescence lifetimes [12], in photon statistics [13], in the resonance fluorescence [1419], in plasmon-induced quantum interference effects [20-22], in the control over population dynamics [23 26], and over nonlinear optical processes [27 34].

In this paper, we theoretically investigate the resonance fluorescence spectrum (RFS) of a hybrid system consisting of a three-level $\Lambda$-type quantum emitter coupled to a MNP which is taken as a gold nanosphere. This work is related to previous works carried out by us [17] and other authors [14 16, 18, 19] on the spectral properties of the spontaneous photons produced in externally driven quantum systems coupled to a metallic nanostructure. These studies revealed the possibility of changing the linewidth and location of the different sidebands of the spectra of the spontaneous photons arising from the back-action of the electric field generated by the metallic nanostructure on the quantum system (exciton-plasmon coupling). Our work presents a new addition in this area and new effects are identified.

We focus in two situations to analyze the RFS of the hybrid system. In the first one we consider the system to be singly driven on exact resonance along one of the optical transitions. We show that the development of the spectrum depends on the relative orientation of the dipole moments of the optical transitions in relation to the nanoparticle. In addition, we demonstrate how the location of the sidebands are strongly modified by the exciton-plasmon coupling. We also show, for the first time, that in the low driving regime it is possible to obtain an ultranarrow spectral line. In the second situation, we consider the system to be, again, singly driven but this time out of resonance, in which case we show the tunability of the center and the width of the spectral line along the non-driven transition. In addition, we show that the photons produced along the undriven transition show strong antibunching. We present a physical description using the dressed states picture showing that both the width and spectral location of the Raman photons are fully captured in the 
secular approximation. These results may be used for creating tunable source of photons which can be used for a probabilistic entanglement scheme which is of interest in the field of quantum information processing.

The paper is organized as follows: Section I I establishes the model, i.e. the Hamiltonian of the system and the main dissipation processes which are needed to derive the time-evolution equations of the quantum system's operators, assuming the rotating wave approximation. We also present the basics for determining the spectral properties of the fluorescent photons through the analysis of the RFS in the steady-state regime. Section III presents numerical results along with relevant discussion. Finally, Section [IV summarizes the main findings of the paper and also presents a discussion on the experimental implementation of our scheme.

\section{THEORETICAL MODEL}

Let us consider a three-level quantum system of the $\Lambda$-type located in close proximity to a MNP. The states of the system are labeled as $|1\rangle,|2\rangle$, and $|3\rangle$ (see Fig. 1).

The Hamiltonian that governs the dynamics of the quantum system can be expressed as

$$
H=H_{A}+H_{F}+H_{\text {Int }}+H_{\text {ext }} .
$$

The Hamiltonian $H_{A}$ of the system reads

$$
H_{A}=\sum_{j=1}^{3} E_{j} \sigma_{j j},
$$

where $E_{j}=\hbar \omega_{j}$ is the energy of the $\mathrm{j}$-th state and $\sigma_{i j}$ are the Pauli operators.

$H_{F}$ is the Hamiltonian of the medium-assisted electromagnetic field

$$
H_{F}=\int d \vec{r} \int_{0}^{\infty} d \omega \hbar \omega \vec{f}_{\lambda}^{\dagger}(\vec{r}, \omega) \overrightarrow{f_{\lambda}}(\vec{r}, \omega),
$$

and it is expressed in terms of a set of bosonic fields $\overrightarrow{f_{\lambda}}(\vec{r}, \omega)$, where $\overrightarrow{f_{\lambda}^{\dagger}}(\vec{r}, \omega)$ plays the role of variable of the electromagnetic field and the medium, including a reservoir associated with the losses in the medium. The field operators obey the usual commutation rules

$$
\left[\overrightarrow{f_{\lambda}}(\vec{r}, \omega), \vec{f}_{\lambda}^{\dagger \dagger}(\vec{r}, \omega)\right]=\delta_{\lambda \lambda^{\prime}} \delta\left(\omega-\omega^{\prime}\right) \delta\left(\vec{r}-\vec{r}^{\prime}\right)
$$

The interaction Hamiltonian is $H_{\text {Int }}$

$$
H_{\text {Int }}=-\int_{0}^{\infty} d \omega[\vec{d} \cdot \vec{E}(\vec{r}, \omega)+H . c .],
$$


(a)

(b)
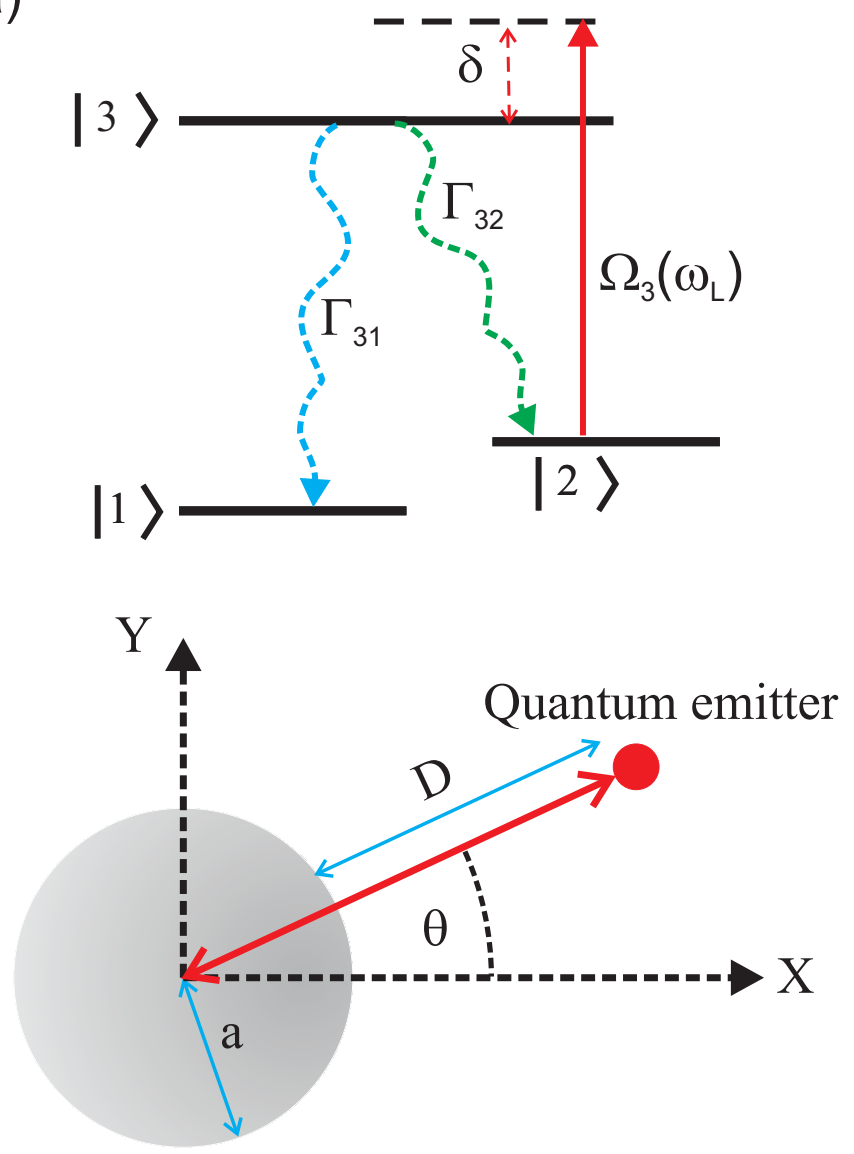

FIG. 1. Three-level scheme illustrating the ground and excited states. Transition $|2\rangle \leftrightarrow|3\rangle$ is driven by a laser field polarized along the $X$ axis with angular frequency $\omega_{L}$ and Rabi frequency $\Omega_{3}$. (b) The quantum system is located at a distance $D$ from the boundary of a nanosphere whose radius is $a$. The dipole moments $\vec{\mu}_{23}$ and $\vec{\mu}_{13}$ are oriented along the $X$ and $Y$ axis, respectively. $\theta$ is the angle with the $X$-axis of the line joining the MNP's center and the QD's center.

$\vec{d}$ being the electric dipole moment operator, which is given by

$$
\vec{d}=\vec{\mu}_{13} \sigma_{13}+\vec{\mu}_{23} \sigma_{23},
$$

and $\vec{E}(\vec{r}, \omega)$ is the field operator (excluding the external driving field) which is defined through [35]

$$
\vec{E}(\vec{r}, \omega)=i \sqrt{\frac{\hbar}{\pi \epsilon_{0}}} \frac{\omega^{2}}{c^{2}} \int d \overrightarrow{r^{\prime}} \sqrt{\epsilon_{I}\left(\overrightarrow{r^{\prime}}, \omega\right)} \stackrel{\leftrightarrow}{G}\left(\vec{r}, \overrightarrow{r^{\prime}}, \omega\right) \vec{f}\left(\overrightarrow{r^{\prime}}, \omega\right)
$$

where $\stackrel{\leftrightarrow}{G}\left(\vec{r}, \overrightarrow{r^{\prime}}, \omega\right)$ is the dyadic Green's tensor. Here, $\epsilon\left(\overrightarrow{r^{\prime}}, \omega\right)=\epsilon_{R}\left(\overrightarrow{r^{\prime}}, \omega\right)+\epsilon_{I}\left(\overrightarrow{r^{\prime}}, \omega\right)$ stands for the complex permittivity. 
Finally, $H_{\text {ext }}$ is the part of the Hamiltonian which accounts for the external coherent coupling and is defined through

$$
H_{e x t}=\hbar \Omega_{3} \sigma_{23} e^{-i \omega_{L} t}+\text { H.c. },
$$

with the effective Rabi field $\Omega_{3}=\vec{\mu}_{23} \cdot \vec{E}_{\text {pump }}\left(\vec{r}_{A}\right) / 2 \hbar$. Here, $\vec{r}_{A}$ denotes the position of the quantum emitter in relation to the MNP $\left(R=\left|\vec{r}_{A}\right|\right)$. The pump field contains the direct pumping field term plus the scattered field from the MNP

$$
\vec{E}_{\text {pump }}\left(\vec{r}_{A}, \omega_{L}\right)=\vec{E}_{0}\left(\vec{r}_{A}, \omega_{L}\right)+\int_{V_{M N P}} d \overrightarrow{r^{\prime}}\left(\epsilon_{m}\left(\omega_{L}\right)-1\right) \stackrel{\leftrightarrow}{G}\left(\vec{r}_{A}, \vec{r}^{\prime}, \omega_{L}\right) \vec{E}_{0}\left(\vec{r}^{\prime}, \omega_{L}\right),
$$

where $\vec{E}_{0}\left(\vec{r}_{A}, \omega_{L}\right)=\frac{1}{2} \hat{u}_{x} E_{0} e^{-i \omega_{L} t}+$ c.c. is the incident field operator and $\hat{u}_{x}$ is the unitary vector along the $X$ axis, and $\epsilon_{m}\left(\omega_{L}\right) / V_{M N P}$ stands for the dielectric constant/volume of the MNP at the frequency $\omega_{L}$. Therefore, the driving field only couples transition $|2\rangle \leftrightarrow|3\rangle$. Note that for an intense incident driving field $\left(\vec{E}_{0}\left(\vec{r}, \omega_{L}\right)\right)$ it can be treated as a c-number, so that the effective Rabi field can be expressed as

$$
\Omega_{3}=\Omega_{3}^{0}\left[1+\int_{V_{M N P}} d \overrightarrow{r^{\prime}}\left(\epsilon_{m}\left(\omega_{L}\right)-1\right) \hat{u}_{x} \stackrel{\leftrightarrow}{G}\left(\vec{r}_{A}, \vec{r}^{\prime}, \omega_{L}\right) \hat{u}_{x}\right] \equiv \Omega_{3}^{0} F_{e}^{x},
$$

$F_{e}^{x}$ being the field enhancement factor. In the above equation $\Omega_{3}$ is the renormalized Rabi frequency associated with the driving field and the field produced by the MNP which acts back upon the quantum emitter, and $\Omega_{3}^{0} \equiv \frac{\mu_{23} E_{0}}{2 \hbar}$ stands for the free space Rabi frequency, i.e., the Rabi frequency which would drive the quantum emitter in the absence of the MNP. Note that the value of $F_{e}^{x}$ will depend on $\theta$, i.e., the relative orientation of the incident field with regard to the axis of the hybrid system.

The bath operators can be traced out in order to obtain a master equation for the reduced quantum system, which enables the derivation of the time evolution of an arbitrary quantum system operator $Q(t)$ which reads

$$
\begin{aligned}
\frac{\partial\langle Q(t)\rangle}{\partial t}= & \frac{i}{\hbar}\left[H_{A}+H_{e x t}, Q\right]-\frac{\Gamma_{31}^{p}}{2}\left(\sigma_{31}\left[\sigma_{13}, Q\right]-\left[\sigma_{31}, Q\right] \sigma_{13}\right) \\
& -\frac{\Gamma_{32}^{p}}{2}\left(\sigma_{32}\left[\sigma_{23}, Q\right]-\left[\sigma_{32}, Q\right] \sigma_{23}\right) \\
& -\frac{\Gamma_{12}}{2}\left(\sigma_{21}\left[\sigma_{12}, Q\right]-\left[\sigma_{21}, Q\right] \sigma_{12}\right) \\
& -\frac{\Gamma_{21}}{2}\left(\sigma_{12}\left[\sigma_{21}, Q\right]-\left[\sigma_{12}, Q\right] \sigma_{21}\right) \\
& -\frac{\gamma_{22}}{2}\left(\sigma_{22}\left[\sigma_{22}, Q\right]-\left[\sigma_{22}, Q\right] \sigma_{22}\right)
\end{aligned}
$$


Here, $\Gamma_{3 j}^{p}$ stands for the spontaneous emission of the quantum emitter modified by the presence of the MNP [20], and they are explicitly given by

$$
\begin{aligned}
& \Gamma_{32}^{p}=\frac{\mu_{23}^{2} \omega_{32}^{2}}{c^{2} \hbar \epsilon_{0}} \hat{u}_{x} \cdot \operatorname{Im}\left[\stackrel{\leftrightarrow}{G}\left(\vec{r}_{A}, \vec{r}_{A}, \omega_{31}\right)\right] \cdot \hat{u}_{x}, \equiv \Gamma_{x}^{p}, \\
& \Gamma_{31}^{p}=\frac{\mu_{13}^{2} \omega_{31}^{2}}{c^{2} \hbar \epsilon_{0}} \hat{u}_{y} \cdot \operatorname{Im}\left[\stackrel{\leftrightarrow}{G}\left(\vec{r}_{A}, \vec{r}_{A}, \omega_{32}\right)\right] \cdot \hat{u}_{y}, \equiv \Gamma_{y}^{p}
\end{aligned}
$$

where $\stackrel{\leftrightarrow}{G}\left(\vec{r}_{A}, \vec{r}_{A}, \omega_{3 j}\right)$ is the electromagnetic Green's tensor evaluated at the frequency $\omega_{3 j}$.

The dissipative process described by the terms with the pre-factors $\Gamma_{21}, \Gamma_{12}$ and $\gamma_{22}$ accounts for the lower levels' repumping and dephasing and we assume that they are uncoupled with the localized surface plasmons due to the low values of the energy splitting $\left(\omega_{21}\right)$ considered. It is worth noting that depending on the orientation of the dipole moments of the emitter's transitions, the decay rates could become very different from one another, i.e., the value of $\Gamma_{31}^{p}$ can strongly differ from that of $\Gamma_{32}^{p}$.

The quantum system's and the coherent parts of the Hamiltonian in an appropriate rotating frame read as

$$
H_{q e, c o h}=\hbar \omega_{21} \sigma_{22}+\hbar\left(\delta+\omega_{21}\right) \sigma_{33}+\hbar\left(\Omega_{3} \sigma_{23}+\Omega_{3}^{*} \sigma_{32}\right)
$$

where $\delta=\omega_{32}-\omega_{L}$, denotes the optical detuning, thus the density matrix equations of motion of the system read as

$$
\begin{aligned}
& \frac{\partial \rho_{22}}{\partial t}=-\Gamma_{21} \rho_{22}-\Gamma_{12}\left(1-\rho_{22}-\rho_{33}\right)+\Gamma_{32}^{p} \rho_{33}-i \Omega_{3} \rho_{32}+i \Omega_{3}^{*} \rho_{23}, \\
& \frac{\partial \rho_{33}}{\partial t}=-\left(\Gamma_{31}^{p}+\Gamma_{32}^{p}\right) \rho_{33}+i \Omega_{3} \rho_{32}-i \Omega_{3}^{*} \rho_{23}, \\
& \frac{\partial \rho_{21}}{\partial t}=-F_{12} \rho_{21}-i \Omega_{3} \rho_{31} \\
& \frac{\partial \rho_{23}}{\partial t}=-F_{32} \rho_{23}-i \Omega_{3}\left(\rho_{33}-\rho_{22}\right) \\
& \frac{\partial \rho_{31}}{\partial t}=-F_{13} \rho_{31}-i \Omega_{3}^{*} \rho_{21} .
\end{aligned}
$$

In writing the above we made use of the following abbreviations: $F_{12}=1 / 2\left(\Gamma_{21}+\Gamma_{12}+\right.$ $\left.\gamma_{22}\right)+i \omega_{21}, F_{32}=1 / 2\left(\Gamma_{31}^{p}+\Gamma_{32}^{p}+\Gamma_{21}+\gamma_{22}\right)-i \delta$, and $F_{13}=1 / 2\left(\Gamma_{31}^{p}+\Gamma_{32}^{p}+\Gamma_{12}\right)+i\left(\delta+\omega_{21}\right)$.

We define the following vector $U(t)=\left[\rho_{22}(t), \rho_{33}(t), \rho_{21}(t), \rho_{12}(t), \rho_{23}(t), \rho_{32}(t), \rho_{31}(t), \rho_{13}(t)\right]^{T}$, where $T$ stands for transpose. Then, we can write Eq. (15) in matrix form as

$$
\frac{d}{d t} U(t)=M U(t)+B
$$


with $M$ being an $(8 \times 8)$ matrix and $B$ a column vector whose coefficients can be determined from Eq. (15). Steady-state values for populations and coherences are derived through $U(\infty)=M^{-1}(-B)$.

We are interested into the spectral properties of the fluorescent photons, in particular the RFS of the hybrid system. In the steady-state regime, this spectrum is proportional to the Fourier transformation of the correlation function $\lim _{t \rightarrow \infty}\left\langle\vec{E}^{-}\left(\vec{r}, t^{\prime}+t\right) \cdot \vec{E}^{+}(\vec{r}, t)\right\rangle$, where $\vec{E}^{-}(\vec{r}, t) / \vec{E}^{+}(\vec{r}, t)$ is the negative/positive frequency part of the radiation field in the far zone. The radiation field consists of a free-field operator and a source-field operator that is proportional to the polarization operator [36]. Therefore, the steady-state RFS can be expressed in terms of the correlation function

$$
S(\omega)=\Re\left[\lim _{t \rightarrow \infty} \int_{0}^{\infty}\left\langle\vec{E}^{-}\left(\vec{r}, t^{\prime}+t\right) \cdot \vec{E}^{+}(\vec{r}, t)\right\rangle e^{-i \omega t^{\prime}} d t^{\prime}\right],
$$

where $\Re[$ ] denotes the real part of the magnitude enclosed in square brackets. In the far-field zone $\left(|\vec{r}| \gg c / \omega_{3 j}, j=1,2\right)$

$$
\vec{E}^{-}(\vec{r}, t)=\frac{\omega_{31}^{2}}{c^{2}|\vec{r}|} \vec{\mu}_{13} \sigma_{31}(t-|\vec{r}| / c)+\frac{\omega_{32}^{2}}{c^{2}|\vec{r}|} \vec{\mu}_{23} \sigma_{32}(t-|\vec{r}| / c),
$$

and $\vec{E}^{+}(\vec{r}, t)=\left(\vec{E}^{-}(\vec{r}, t)\right)^{\dagger}$. We will assume that $\omega_{31} \approx \omega_{32}$. In what follows we consider that the following condition holds: $\vec{\mu}_{13}=\mu \hat{u}_{y}$, and $\vec{\mu}_{23}=\mu \hat{u}_{x}$, whereas the direction of detection of the fluorescent field is perpendicular to the plane $X Y$ which contains the electric dipole moments $\vec{\mu}_{13}$ and $\vec{\mu}_{23}$. Substituting Eq. (18) into Eq. (17) results into an expression which contains two-time correlation functions. The calculation of $S(\omega)$ can be performed by means of the quantum regression theorem [36, 37] (see Appendix A for details). The RFS given in Eq. (17) has two contributions: one of them accounts for the photons produced along the $|1\rangle \leftrightarrow|3\rangle$ transition (the term involving $\hat{U}_{13}(\tau)$ as defined in Appendix (A), while the other is related with the photons produced along the $|2\rangle \leftrightarrow|3\rangle$ transition (the term involving $\left.\hat{U}_{23}(\tau)\right)$. In writing Eq. (17) and in the rest of this work, we abbreviate $\omega-\omega_{L}$ by $\omega$, but we should interpret $\omega$ as a frequency measured relative to the laser frequency $\omega_{L}$ since we will assume that the hybrid system is singly driven by $\Omega_{3}$.

The statistical properties of the fluorescent photons can be determined through the normalized second-order correlation function (intensity-intensity correlation) of the fluorescent signal emitted by the hybrid system which is given by 


$$
g^{(2)}(\vec{r}, t, \vec{r}, t+\tau)=\frac{G^{(2)}(\vec{r}, t, \vec{r}, t+\tau)}{G^{(1)}(\vec{r}, t) G^{(1)}(\vec{r}, t+\tau)} .
$$

The first- and second-order correlation functions appearing in Eq. (19) can be expressed in terms of the positive and negative frequency parts of the electric field operators as

$$
\begin{aligned}
& G^{(1)}(\vec{r}, t)=\left\langle\vec{E}^{-}(\vec{r}, t) \vec{E}^{+}(\vec{r}, t)\right\rangle, \\
& G^{(2)}(\vec{r}, t)=\left\langle\vec{E}^{-}(\vec{r}, t) \vec{E}^{-}(\vec{r}, t+\tau) \vec{E}^{+}(\vec{r}, t+\tau) \vec{E}^{+}(\vec{r}, t)\right\rangle .
\end{aligned}
$$

We will assume that first- and second-order correlation functions leading to Eq. (19) will be determined under stationary conditions by invoking again the quantum regression theorem.

\section{NUMERICAL RESULTS}

We start by analyzing how the presence of the MNP modifies the spontaneous emission rates of the quantum emitter. The MNP is a gold nanosphere with radius $a$ as depicted in Fig. 1(b). The radius of the nanosphere is taken $a=50 \mathrm{~nm}$. Both the quantum emitter and the MNP are embedded in a lossy free dielectric background with dielectric constant 2.25. The dielectric function of the gold MNP was extracted from optical data [38]. We calculate the spontaneous decay rates and the field enhancement factor via a Green's tensor formalism of the electromagnetic field for a single spherical scatterer for wavelength $\lambda=947$ $\mathrm{nm}$. Namely, the calculation of the electromagnetic field around a spherical scatterer is based on the classical Mie theory [39]. The corresponding EM Green's tensor is calculated within the same framework, i.e., Mie theory, and the corresponding formalism can be found elsewhere [40].

Figure 2(a) show the radiative decay rates $\Gamma_{x}^{p}$ and $\Gamma_{y}^{p}$ modified by the presence of the MNP as a function of the distance $D$ between the surface of the nanosphere to the quantum emitter. The external driving field was selected to be parallel to the $X$ axis. A close inspection of Fig. 2(a) shows a highly distinctive behaviour for the two decay rates: The $\Gamma_{x}^{p}$ decay rate shows a non-monotonous variation versus the distance $D$ around the free space value $\left(\Gamma_{0}\right)$, whereas the $\Gamma_{y}^{p}$ decay rate exhibits a large variation taking values up to one order of magnitude greater than the free space value. This asymmetric behavior results in 
privileging the decay from the upper state $|3\rangle$ to the lower state $|1\rangle$ in comparison with the decay from $|3\rangle$ to the lower state $|2\rangle$, and originates from the difference between the longitudinal surface plasmon resonance along the $Y$-axis and the transverse surface plasmon resonance along the $X$-axis. This indicates that the MNP acts as a nanoscale cavity which enhances the strength of the vacuum fluctuations depending on the distance $D$ and the orientation of the dipoles of the system.

In Fig. 2(b) we present the results for the field enhancement factor $F_{e}^{x}$ in the case where the hybrid system is singly driven, for two different values of the angle $\theta$. For $\theta=0$ (dasheddotted curve) we observe that the effective field felt by the QD is reduced with regard to the free space value and exhibits a minimum at a very short distance. On the other hand, for $\theta=90^{\circ}$ (solid curve) the effective field is enhanced and exhibits a monotonous decrease for increasing values of $D$. Based on this, we can selectively suppress or enhance a given spontaneous emission channel of the quantum emitter by varying the distance $D$ and/or the orientation of the dipole moment. Besides spontaneous emission, one can also modify the corresponding Rabi frequency of the emitter by varying the same parameters of the hybrid system. This anisotropic resonant phenomenon is the key idea that will allow to modify the spectral properties of the fluorescent photons produced by the hybrid system.
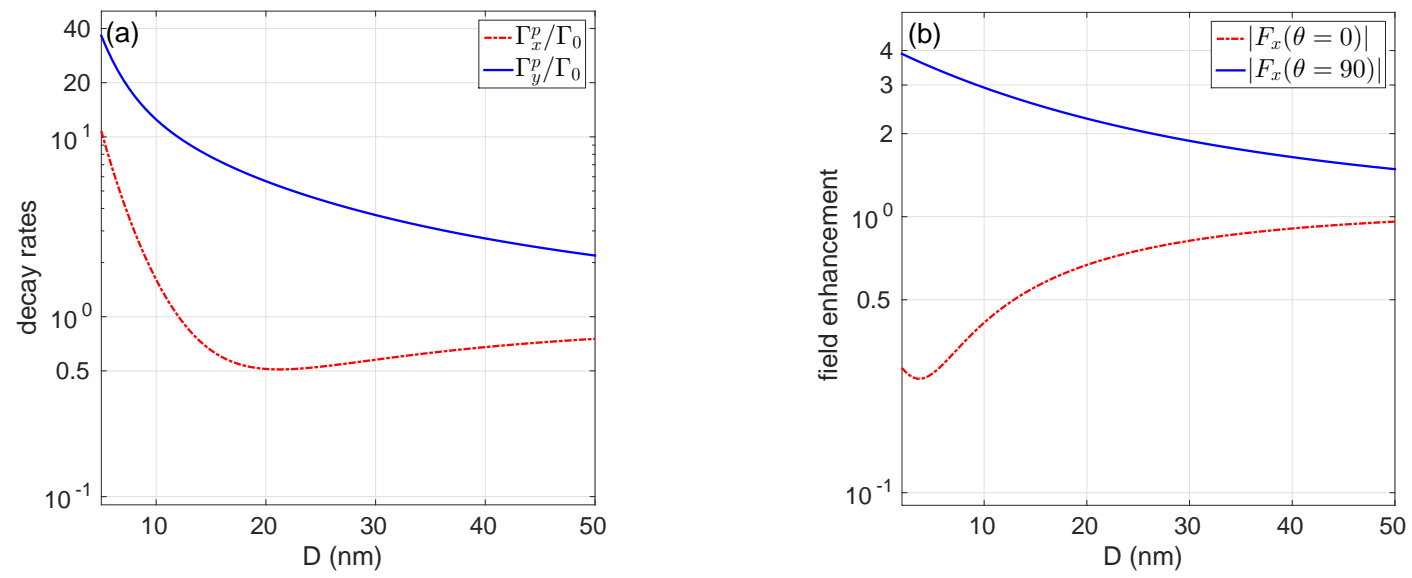

FIG. 2. (a) Plasmon modified radiative decay rates of the quantum emitter $\Gamma_{x}^{p}$ (dashed-dotted curve), and $\Gamma_{y}^{p}$ (solid curve) in units of $\Gamma_{0}$ vs the distance $D$ in nm. (b) Field enhancement factor $\left(\left|F_{e, x}\right|\right)$ vs distance $D$ when the angle is $\theta=0^{0}$ (dashed-dotted curve), and $\theta=90^{\circ}$ (solid curve).

Next, we examine the influence of the MNP on the steady state RFS. Numerical results for the RFS are obtained through Eq. (17). The results are displayed in Fig. 3(a) for 
a Rabi frequency of $\Omega_{3}^{0}=2.5 \Gamma_{0}$ : this value is selected in the range which shows the full development of the spectral features for the isolated quantum emitter. The driving field is considered to be on resonance $(\delta=0)$. The solid curve corresponds to the spectrum obtained for the isolated quantum emitter and consists of a central peak, a red detuned sideband as well as three blue detuned sidebands. For the hybrid system we choose $D=20$ nm between the quantum emitter and the MNP: the dashed curve is obtained when $\theta=0$ and the dashed dotted curve is for $\theta=90$. In the case with $\theta=0$, we observe that for this geometrical configuration the RFS collapses to a nearly single central peak which is broader than that obtained in the absence of the MNP whereas the far detuned blue sidebands remain unresolved, i.e., the spectral separation of these two sidebands is lesser than their HWHM. These results can be explained in light of the effect of the MNP on the effective field which drives the system: according to dashed-dotted curve in Fig. 2(b) the value for $\left|F_{e, x}\right|$ is less than unity for this gap distance $\left(\left|\Omega_{3}\right|<\left|\Omega_{3}^{0}\right|\right)$. The RFS changes dramatically when considering the other configuration $(\theta=90)$, where the presence of the MNP results in $\left|F_{e, x}\right|>1$, leading to the progressive separation of the sidebands. At the same time the sidebands broaden as a consequence of the modification of the decay rates by the plasmonic interaction. It is expected that for this particular geometrical configuration, the closer the MNP to the quantum emitter, the greater the separation of the sidebands from the central peak.

The appearance of a quintuplet in the RFS arises from the fact that there are two contributions to the RFS as indicated in Eq. (18). The first contribution arises from the photons produced along the transition $|3\rangle \leftrightarrow|2\rangle$ (see dashed curve in Fig. [3(b)): this transition is responsible for the emergence of the central peak and two sidebands simmetrically located around $\omega=0$, and mimics the Mollow triplet of a two-level system. The second contribution arises from the photons produced along the undriven transition $|3\rangle \leftrightarrow|1\rangle$ (see solid curve in Fig. 3(b)): here we observe the lack of a central peak for this channel which should be expected to appear at $\omega=\omega_{L}+\omega_{21}$ in the case that transition $|3\rangle \leftrightarrow|1\rangle$ would have been driven by an external laser field. Here we also observe the emergence of two additional blue detuned sidebands.

We carried out an analysis in the dressed state picture (DSP) of the RFS for the current situation (the details are provided in Appendix $(\mathrm{B})$ : there we show that in the secular approximation, which consists in obtaining equations of evolution for populations and coherences 

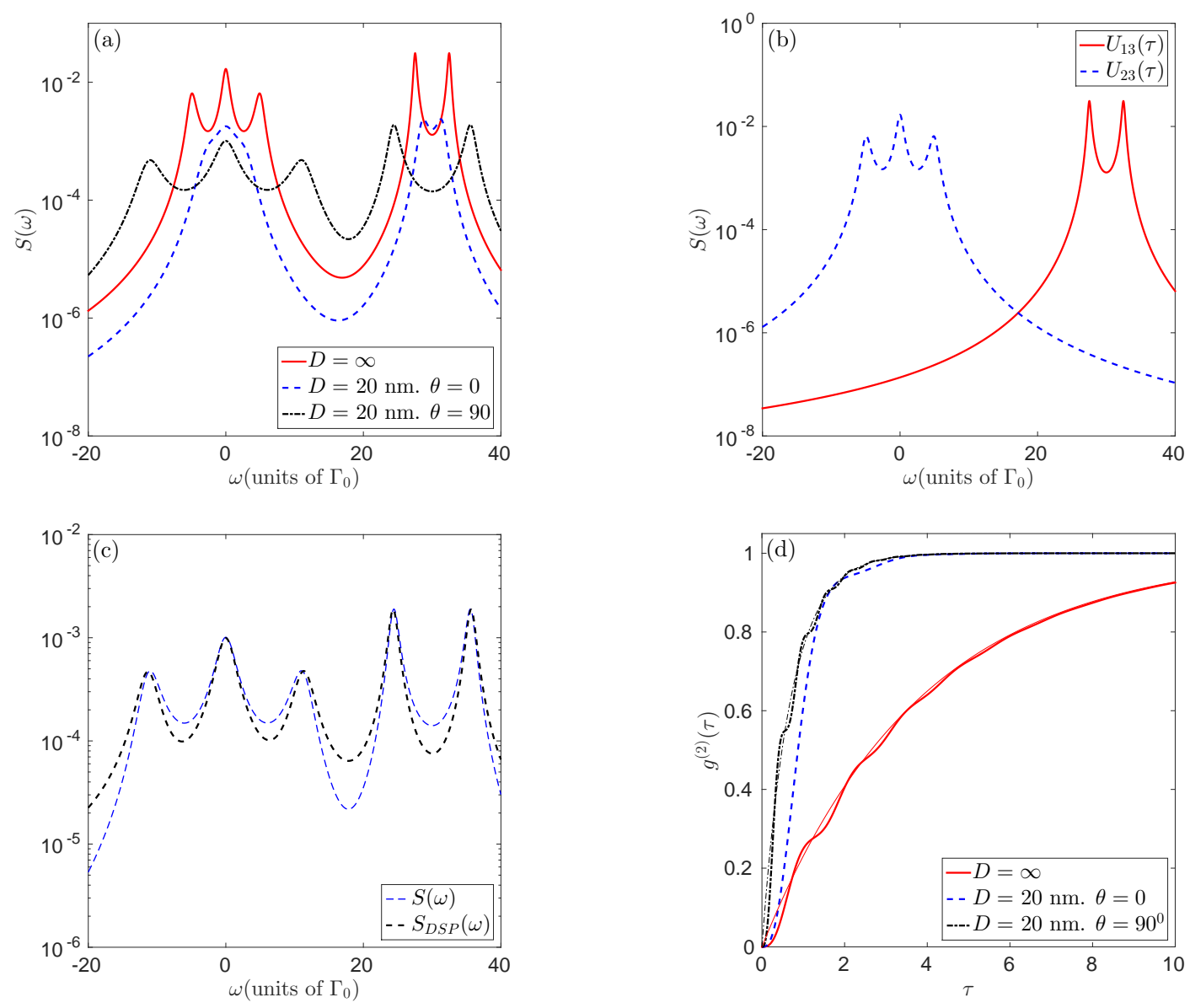

FIG. 3. (a) Steady-state RFS $(S(\omega))$ of the system driven on resonance $\delta=0$ when $\Omega_{3}^{0}=2.5 \Gamma_{0}$, in the absence of MNP (solid curve) and with a distance $D=20 \mathrm{~nm}$ : dashed curve when $\theta=0$, and dashed-dotted curve for the case with $\theta=90^{\circ}$. (b) Contributions to RFS of correlations $U_{13}(\tau)$ (solid curve) and $U_{24}(\tau)$ (dashed curve) for the isolated quantum emitter. (c) Full RFS $\left(S(\omega)\right.$, thin dashed curve) and RFS computed in the DSP $\left(S_{D S P}(\omega)\right.$, thick dashed curve) for $\Omega_{3}^{0}=2.5 \Gamma_{0}, D=20 \mathrm{~nm}$, and $\theta=90^{\circ}$. (d) Time evolution of intensity-intensity correlation $\left(g^{(2)}(\tau)\right)$ for the fluorescent photons produced along transition $|1\rangle \leftrightarrow|3\rangle$ in the cases considered in panel (a). Thin solid curves are the corresponding normalized second-order correlations computed in the DSP. $\tau=t \Gamma_{0}$ stands for normalized time. Other numerical values used are: $\omega_{21}=30 \Gamma_{0}$, $\Gamma_{12}=\Gamma_{21}=0.008 \Gamma_{0}, \gamma_{22}=0.0014 \Gamma_{0}$.

while neglecting the couplings between them, the RFS spectrum can be decomposed as a sum of Lorentzians whose peaks and effective widths are obtained in terms of the physical decays (see Eqs. (묘 $)-(\bar{B} 9))$ and the coefficients of expansion of the dressed states in terms of 
the bare states $\left(c_{2}\right.$ and $\left.c_{3}\right)$. This analysis explains the location and heights of the Lorentzians found in Fig. 3. As an example of this statement we compare in Fig. 31(c) the RFS computed in the DSP using Eq. (B7) (thick dashed curve) with the one obtained via the full solution (thin dashed curve) using Eq. (17), which reveals the good agreement between them. According to this analysis, the far detuned blue sidebands are located at $\lambda_{+}$, and $\lambda_{-}$, which originate from transitions from dressed levels $|+, N\rangle \leftrightarrow|1, N-1\rangle$ and $|-, N\rangle \leftrightarrow|1, N-1\rangle$, $\Gamma_{+1}$ and $\Gamma_{-1}$ being the corresponding widths, respectively ( $N$ stands for the number of photons in each manifold). The sidebands simmetrically located around $\omega=0$ are produced by transitions from dressed levels $|+, N\rangle \leftrightarrow|-, N-1\rangle$ and $|-, N\rangle \leftrightarrow|-, N-1\rangle$, $\Gamma_{+-}$being the width for both of them. Finally, the central peak is produced from transitions between the same levels of adjacent manifolds: $|+, N\rangle \leftrightarrow|+, N-1\rangle$ and $|-, N\rangle \leftrightarrow|-, N-1\rangle$.

The statistical properties of the fluorescent photons can be determined through the analysis of the time evolution of the normalized second-order correlation function given in Eq. (19). Here we assume that selective detection of the fluorescent photons is performed, thus we only have to deal with photons produced along the undriven transition $|1\rangle \leftrightarrow|3\rangle$. The resuls are depicted in Fig. 3(d) for the same cases considered in Fig. 3)(a). Here, we observe that such fluorescent photons remain in the anti-bunching regime within the time interval depicted for the isolated quantum emitter (solid curve), whereas the reaching to the level of unity (the crossing being the bunching regime) is accelerated in the presence of the MNP. This speed up to reach the bunching regime is expected since the presence of the MNP results in changing the decay rates and the electric field acting upon the quantum emitter. The thin solid curves appearing in Fig. 3(d) are the corresponding normalized intensityintensity correlations computed in the DSP, and show a good agreement to predict the time evolution under such driving condition.

It is well known that the development of the spectral features in the RFS is field $\left(\Omega_{3}\right)$ dependent. Now we assume that the hybrid system is singly driven with a Rabi frequency below saturation. In this case the secular approximation used to derive the RFS in the DSP does not hold, thus we resort to numerical results for the RFS in the bare state basis. We consider a distance of $D=20 \mathrm{~nm}$. We also assume a polarization selective detection for the fluorescent photons along the channel $|3\rangle \leftrightarrow|1\rangle$, thus we only take into account the signal associated to the correlation $U_{13}(\tau)$, i.e., $S_{13}(\omega)$ as defined in Appendix $\mathrm{A}$. The spectra obtained for different Rabi frequencies are depicted in Fig. 4(a)-(b). There, we 
observe that the spectra exhibit subnatural linewidths for such low values of the driving field. The spontaneous decay rates for this situation are $\Gamma_{x}^{p}=0.51 \Gamma_{0}$, and $\Gamma_{y}^{p}=5.67 \Gamma_{0}$ for the selected gap distance. Nevertheless, the HWHM of the spectral feature in Fig. 4 (a) is $0.06 \Gamma_{0}$ for the isolated quantum emitter, whereas it changes to $0.02 \Gamma_{0}\left(0.09 \Gamma_{0}\right)$ in the case with $\theta=0(\theta=90)$. A similar trend is obtained for the case depicted in Fig. 4(b) where the HWHM is $0.21 \Gamma_{0}$ in the absence of MNP, and it changes to $0.04 \Gamma_{0}\left(0.31 \Gamma_{0}\right)$ in the case with $\theta=0(\theta=90)$. The emergence of this narrow spectral feature in the spectrum arises from the collapse of the two spectral peaks obtained in Fig. 3(b) (solid curve) into a single peak. Thus, the case with $\theta=0$ is the most favourable to obtain a narrower spectrum, whose ultimate origin relies in the lesser than unity field enhancement factor obtained for $\theta=0$. The previous results indicate that the filtered RF photons along the non-driven transition are mostly liberated from the transitions properties and are tailored by the field enhancement of the MNP.

The time evolution of the normalized second-order correlation function is also depicted in Fig. 4(c) for one of the Rabi frequencies in the low driving regime. There we can devise that this correlation remain also in the anti-bunching regime for a large period of time in the case with $\theta=0$, which is fully attributable to the fact that the field enhancement factor is less than unity, whereas it is accelerated when $\theta=90$ (where $\left|F_{e, x}\right|>1$ ) compared to the case of the isolated quantum emitter. The slow down(speed up) of $g^{(2)}(\tau)$ reflects in the time domain the narrowing(broadening) of this spectral feature with regard to the case of the isolated emitter (solid curve in Fig. 4(c)).

Up to now we have assumed that the driving field is on resonance with the transition $|2\rangle \leftrightarrow|3\rangle$, i.e., $\delta=0$. We now turn our attention to the question of how the RFS is modified when the above transition is driven out of resonance. We will show that photons produced along the transition $|1\rangle \leftrightarrow|3\rangle$ exhibit subnatural linewidth, whose frequency is tunable by changing the sign of $\delta$, the gap distance $(D)$ and the Rabi frequency of the driving field $\left(\Omega_{3}^{0}\right)$. To this end we consider $\theta=90^{0}$ and set $\delta=-30 \Gamma_{0}$. We also assume that a selective detection of the fluorescent photons is applied to isolate the photons produced along the two transition channels. In particular, we are only concerned with the detection of photons produced along transition $|1\rangle \leftrightarrow|3\rangle$, i.e., with $S_{13}(\omega)$ as defined in Eq. (A2). The results obtained when $\Omega_{3}^{0}=2 \Gamma_{0}$ are depicted in Fig. 5)(a) for several interparticle distances: $D=20$ nm (dashed curve), $D=40 \mathrm{~nm}$ (dotted curve), and $D=\infty$ (isolated quantum emitter - 

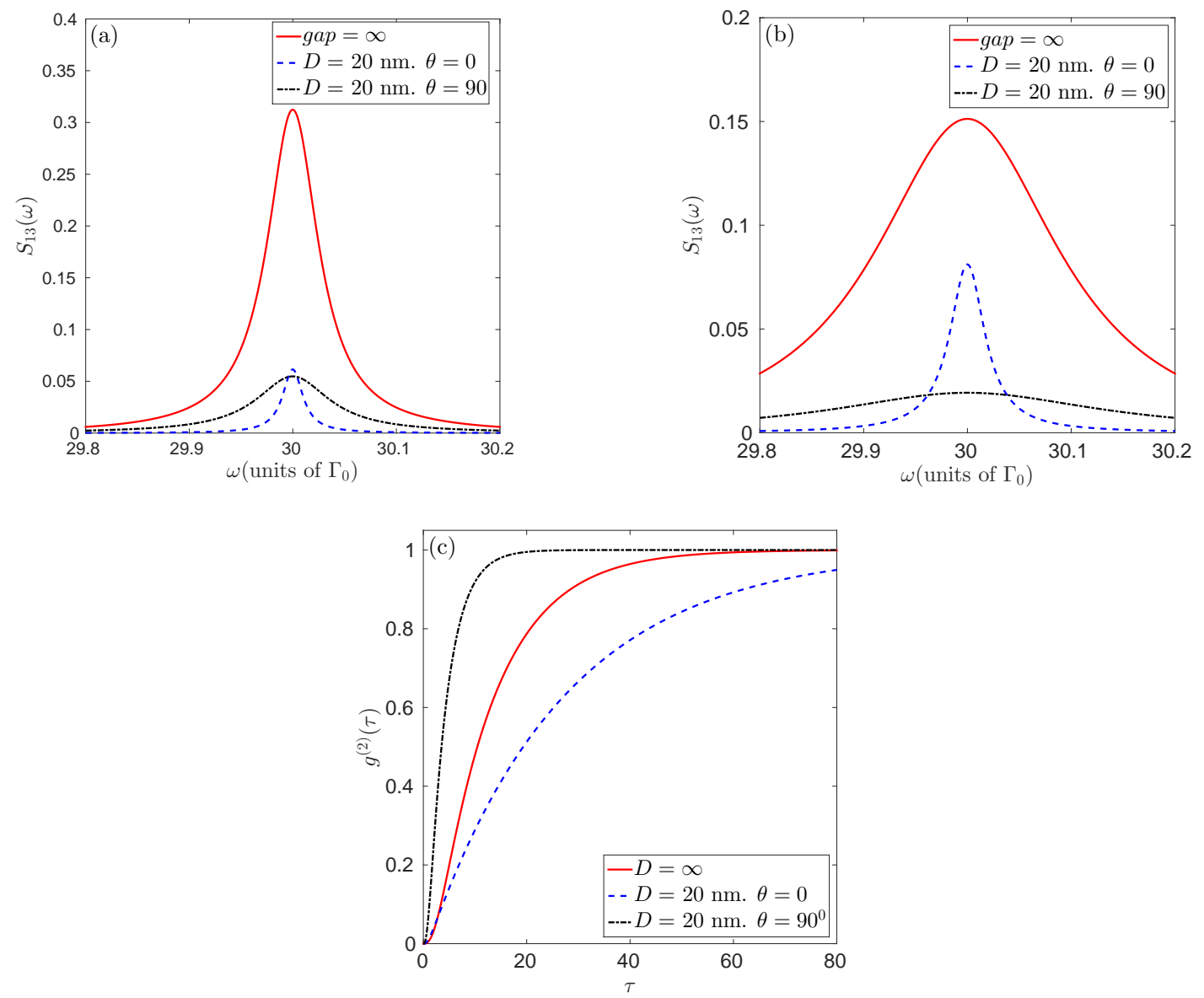

FIG. 4. Steady-state RFS $\left(S_{13}(\omega)\right)$ of the singly driven quantum emitter for different Rabi frequencies: (a) $\Omega_{3}^{0}=0.1 \Gamma_{0}$ and (b) $\Omega_{3}^{0}=0.2 \Gamma_{0}$. (c) Time evolution of normalized second-order correlation function when $\Omega_{3}^{0}=0.2 \Gamma_{0}$. Isolated emitter (solid curve), $\theta=0$ (dashed curve), and $\theta=90$ (dashed-dotted curve). Also, the rest of parameters as in Fig. 3 .

solid curve). Here, we show that the Raman photons are blue detuned with regard to the central peak and that the peak frequency of the Raman photons can be tuned by varying the gap distance between the quantum emitter and the MNP. For the case of the isolated quantum emitter (solid curve), the spectral location of the Raman photons is found to be $30.13 \Gamma_{0}$ apart from $\omega_{L}$. This value changes to $30.67 \Gamma_{0}$ when $D=20 \mathrm{~nm}$, and to $30.36 \Gamma_{0}$ when $D=40 \mathrm{~nm}$. The linewidth $(\mathrm{HWHM})$ of these spectral features are: $0.011 \Gamma_{0}, 0.042 \Gamma_{0}$ and $0.019 \Gamma_{0}$, respectively. We observe that the lower the interparticle distance, the larger the spectral shift, although at the expense of obtaining a small increment of the linewidth.

In the case of driving the hybrid system out of resonance but for a positive detuning, 
the Raman photons are also blue detuned with regard to $\omega_{L}$. This is shown in Fig. [5) for $\delta=+10 \Gamma_{0}$ and $\Omega_{3}=\Gamma_{0}$, with the same interparticle distances $D$ previously considered.

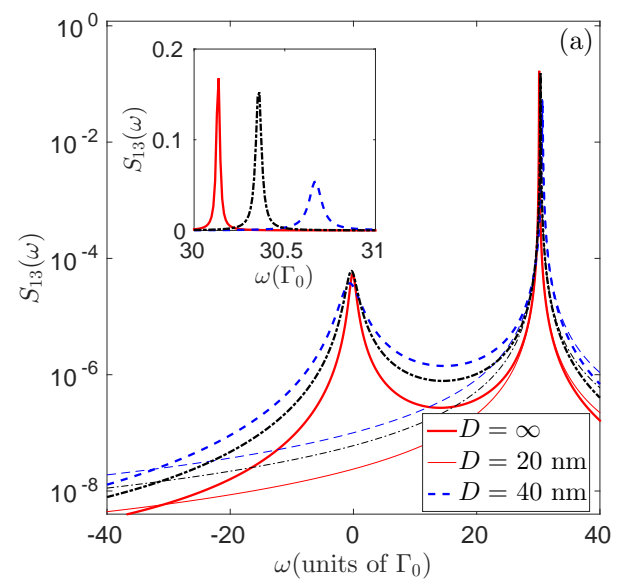

(c)

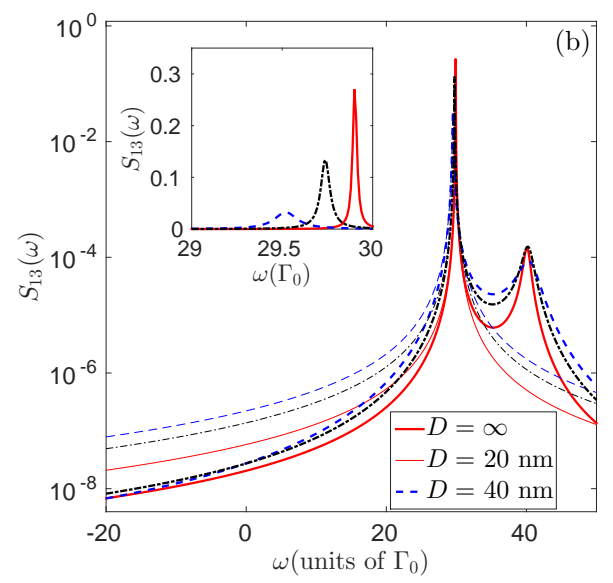

(d)

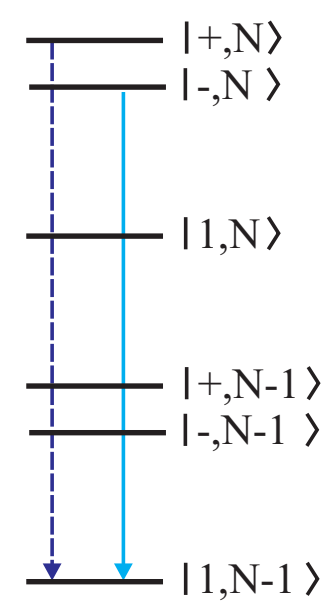

FIG. 5. (a) Steady-state RFS $\left(S_{13}(\omega)\right)$ of the singly driven hybrid system with $\theta=90^{\circ}$ for several interparticle distances: $D=20 \mathrm{~nm}$ (dashed curve), $D=40 \mathrm{~nm}$ (dotted curve), and $D=\infty$ (isolated quantum emitter - solid curve). (a) $\delta=-30 \Gamma_{0}$, and $\Omega_{3}^{0}=2 \Gamma_{0}$ (b) $\delta=+10 \Gamma_{0}$, and $\Omega_{3}^{0}=\Gamma_{0}$. The thin solid curves are the spectral feature associated to the Raman photons determined in the DSP. (c)/(d) Transitions between dressed states accounting for $S_{13}(\omega)$ in the case considered in (a)/(b): the solid vertical arrow indicates the transition responsible for the Raman photons, whereas the dashed vertical arrow stands for the other sideband.

The RFS in the DSP for the Raman photons is obtained by picking the corresponding term in Eq. (B8) associated with transition $|1\rangle \leftrightarrow|3\rangle$ which is labelled as $S_{a}^{D S P}(\omega)$. There 
we observe that this term is the sum of two Lorentzians: one located at $\lambda_{+}$whose width is $\Gamma_{+1}$, and the other located at $\lambda_{-}$and width $\Gamma_{-1}$ (see Eq. (Bי $)$ ). In the case of of $\delta<0$ the Raman photons are obtained through transitions from $|+, N\rangle$ to $|1, N-1\rangle$ as indicated by the solid vertical arrow in Fig. 5(c) which connects two adjacent manifolds in the DSP. On the other hand, in the case with $\delta>0$ the Raman photons are produced along transitions from $|-, N\rangle$ to $|1, N-1\rangle$ as indicated by the solid vertical arrow in Fig. 5 (d). We have checked that the linewidth of the Raman photons $\Gamma_{+1}\left(\Gamma_{-1}\right)$ in the case of $\delta<0(\delta>0)$ coincide with the ones obtained via numerical solution of $S_{13}(\omega)$ in Eq. (A2). The Lorentzian accounting for these transitions are also plotted as thin solid curves in Fig. 5(a)-(b) showing that both the width and spectral location of the Raman photons are fully captured by the description in the dressed basis.

It is worth mentioning that the linewidth of the Raman photons is nearly insensitive to the values of $\Gamma_{x}^{p}$ and $\Gamma_{y}^{p}$, which in turn depend upon the distance $R$. In fact the small broadening of this narrow spectral feature originates mainly from the field enhancement $\left(F_{e, x}\right)$ at the position of the quantum emitter. This point has been confirmed numerically in the following way: we determined the linewidth of the Raman photons while setting $F_{e, x}=1$ (which is an unphysical situation) for the same distances considered in Fig. 5(a). The results obtained show changes in the third decimal place. In other words, the tunability of the Raman photons can be attributed to the field enhancement factor experienced by the quantum emitter due to the presence of the nanoparticle.

Figure 5(a)-(b) indicate us that the spectral location of the Raman photons can be changed through varying either $\delta_{3}, \Omega_{3}^{0}$ and/or the gap distance. The narrowness and tunability of this spectral line is further shown in Fig. 6)(a)-(b). By inspecting these two panels one can identify a tradeoff between the tunability and the linewidth of this spectral line.

The statistical properties of the Raman photons in the case with $\delta<0$ can be determined through the analysis of the time evolution of the normalized second-order correlation function

$$
g_{R-P}^{(2)}(\tau)=\frac{\left\langle\sigma_{+1}(\infty) \sigma_{+1}(\tau) \sigma_{1+}(\tau) \sigma_{1+}(\infty)\right\rangle}{\left\langle\sigma_{+1}(\infty) \sigma_{1+}(\infty)\right\rangle\left\langle\sigma_{+1}(\infty) \sigma_{1+}(\infty)\right\rangle}
$$

which implicitely assumes a selective spectral detection of photons along channel $|+\rangle \leftrightarrow|1\rangle$. The time evolution of magnitude $g_{R-P}^{(2)}(\tau)$ is depicted in Fig. 6(c). The main difference between the intensity-intensity correlation depicted in Fig. 6(c) and those previously considered (see Figs. 3(d) and 4(c)) relies on the fact that here we are dealing with spectrally 

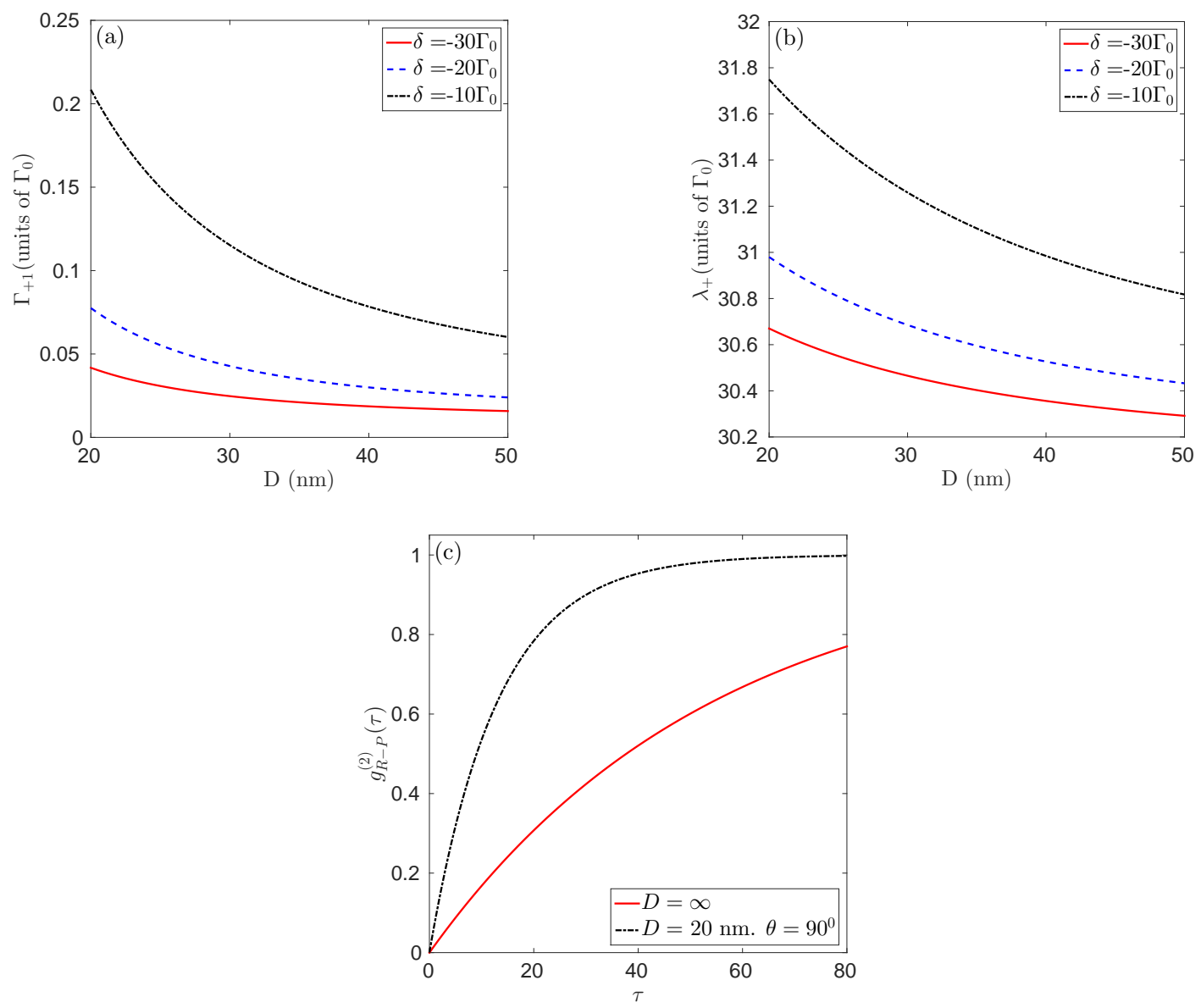

FIG. 6. (a) Linewidth of the Raman photons $\left(\Gamma_{+1}\right)$ versus distance $D$ for different values of the optical detuning $\delta$. (b) Spectral location of the Raman photons $\left(\lambda_{+}\right)$versus distance for different values of the optical detuning $\delta . \delta=-30 \Gamma_{0}$ (solid curve), $\delta=-20 \Gamma_{0}$ (dashed curve), and $\delta=-10 \Gamma_{0}$ (dashed-dotted curve). (c) Time evolution of intensity-intensity correlation for the Raman spectral line: isolated emitter (solid curve), and $D=20 \mathrm{~nm}$ (dashed-dotted curve) with $\theta=90^{0}$.

filtered photons around the Raman line. Anyway, all these correlations exhibit a strong antibunching.

\section{CONCLUSIONS}

In this work we present a theoretical study of the spectral properties of the fluorescent photons produced by a hybrid system consisting of a $\Lambda$-type three-level quantum emitter in close proximity to a MNP. We show that the changes in the effective field felt by the 
quantum emitter and the corresponding modifications of the spontaneous decay rates due to the presence of the MNP manifest themselves as modifications of the width and location of the sidebands of the spectrum. We predict a subnatural linewidth for the narrow spectral line produced along the non-driven transition when the system is singly driven on resonance by a very weak field. In addition, we demonstrate the change in location and width of the Raman peak along the non-driven transition when the system is singly driven out of resonance by a moderate laser field. This tunability can be achieved through varying either the Rabi frequency, the detuning and/or the separation between the quantum emitter and the MNP. We also have studied the statistical properties of the photons along the undriven transition either on resonance and at a very low driving field, and out of resonance. In all cases it is shown that the photons along that transition exhibit a strong antibunching and that the intensity-intensity correlations lacks from the typical oscillatory behavior obtained in a singly driven two-level system [41, 42].

Before closing, we need to pinpoint that the hybrid system investigated in this work can be implemented in realistic semiconductor quantum dot (QD) - MNP systems. The present state of the art of sample growth and coherent-carrier control allows the implementation of QDs with the optical characteristics similarly to the three-level system considered here. A $\Lambda$-type quantum system can be for example established by applying an in-plane magnetic field (in the growth plane) of a InGaAs dots in a GaAs matrix provided that the Zeeman splitting of the exciton states is larger than the linewidths of the optical transitions [43]. The application of such magnetic field, in the so-called Voigt geometry, allows to link states $|2\rangle$ and $|3\rangle$ through a $\pi_{x}$ linearly polarized optical field, whereas whereas $|1\rangle$ and $|3\rangle$ can be driven through a $\pi_{y}$ linearly polarized laser field.Furthermore, a Mollow-like quintuplet spectrum has been experimentally observed in InAs/GaAs quantum dots [44].

The search for semiconductor-based single-photon sources also triggered the study of resonance fluorescence photons in the Heitler regime along with their second order correlation properties [45 47], showing the potential to generate single photons with a bandwidth close to the natural linewidth of the QD transition. Here, we have shown that a weakly driven three-level quantum emitter coupled to a MNP leads to subnatural spectral lines. One advantage of the current hybrid system relies in the fact that the RF photons are produced along the undriven transition, which lacks of the elastic component, while the RF photons produced along the on-resonance driven transition can be removed by using a polarization 
selective detection scheme. The creation of Raman photons along a non-driven transition has been also experimentally addressed in QDs, either in free space [48, 49] or coupled to a cavity [50, 51]. This process has been proposed as a tunable source for a probabilistic entanglement scheme, a property of paramount interest to quantum information processing [48]. Quantum interference between two cw Raman photons as high as 0.98 has also been reported [49]. In addition, hybrid complexes consisting of self-assembled QDs have been grown and covered with metal nanocrystals [52]. We would like to draw the attention to recent work where the controlled coupling of single QDs to a plasmonic nanoantenna has been demonstrated [10, 11, 53 56]. Therefore, the hybrid system considered here could be fabricated using available growth and positioning technologies for the elements involved. 


\section{Appendix A: Appendix A: RFS in the bare state basis}

To determine the RFS in the bare state basis we make use of vector $U(t)$ defined previously in Eq. (16). The evaluation of the two-time correlation functions appearing in Eq. (17) can be recast into

$$
S(\omega)=f^{2}(r)|\mu|^{2} \Re\left[\int_{0}^{\infty}\left(\left\langle\sigma_{31}(\tau) \sigma_{13}(\infty)\right\rangle+\left\langle\sigma_{32}(\tau) \sigma_{23}(\infty)\right\rangle\right) e^{-i \omega \tau} d \tau\right] .
$$

Equation (A1) can be recast as $S(\omega)=S_{13}(\omega)+S_{23}(\omega)$ with

$$
\begin{aligned}
& S_{13}(\omega)=f^{2}(r)|\mu|^{2} \Re\left[\int_{0}^{\infty}\left\langle\sigma_{31}(\tau) \sigma_{13}(\infty)\right\rangle e^{-i \omega \tau} d \tau\right], \\
& S_{23}(\omega)=f^{2}(r)|\mu|^{2} \Re\left[\int_{0}^{\infty}\left\langle\sigma_{32}(\tau) \sigma_{23}(\infty)\right\rangle e^{-i \omega \tau} d \tau\right],
\end{aligned}
$$

where the term $S_{13}(\omega)\left(S_{23}(\omega)\right)$ accounts for the fluorescent photons produced along transition $|3\rangle \leftrightarrow|1\rangle(|3\rangle \leftrightarrow|2\rangle)$.

The two-time correlation functions which appear in Eq. (A1) can be determined with the aid of the quantum regression theorem [36, 37] and the optical Bloch equations, i.e., Eq. (16). To this end we define the column vector

$$
\begin{aligned}
\hat{U}_{j 3}(\tau)= & {\left[\left\langle\sigma_{22}(\tau) \sigma_{j 3}(\infty)\right\rangle,\left\langle\sigma_{33}(\tau) \sigma_{j 3}(\infty)\right\rangle,\right.} \\
& \left\langle\sigma_{12}(\tau) \sigma_{j 3}(\infty),\right\rangle\left\langle\sigma_{21}(\tau) \sigma_{j 3}(\infty)\right\rangle, \\
& \left\langle\sigma_{32}(\tau) \sigma_{j 3}(\infty),\right\rangle\left\langle\sigma_{23}(\tau) \sigma_{j 3}(\infty)\right\rangle, \\
& \left.\left\langle\sigma_{13}(\tau) \sigma_{j 3}(\infty)\right\rangle,\left\langle\sigma_{31}(\tau) \sigma_{j 3}(\infty)\right\rangle\right]^{T},(j=1,2),
\end{aligned}
$$

where the super-index $T$ stands for transpose. According to the quantum regression theorem, for $\tau>0$ the vector $\hat{U}_{j 3}$ satisfies

$$
\frac{d \hat{U}_{j 3}(\tau)}{d \tau}=M \hat{U}_{j 3}(\tau)+B\left\langle\sigma_{j 3}(\infty)\right\rangle,
$$

$M$ being the $8 \times 8$ matrix of the coefficients of Eq. (16) and $B$ the corresponding column vector.

By working in the Laplace space we obtain the steady-state RFS. Specifically we have

$$
\begin{aligned}
S(\omega) \propto & \Gamma_{0} \Re\left\{\sum_{l=1}^{l=8} R_{8, l}\left(i z_{1}\right)\left(\hat{U}_{13}^{(l)}(\infty)+\frac{B_{l}}{i z_{1}}\left\langle\sigma_{13}(\infty)\right\rangle\right)\right. \\
& \left.+\sum_{l=1}^{l=8} R_{5, l}\left(i z_{1}\right)\left(\hat{U}_{23}^{(l)}(\infty)+\frac{B_{l}}{i z_{1}}\left\langle\sigma_{23}(\infty)\right\rangle\right)\right\},
\end{aligned}
$$


where $\hat{U}_{j 3}^{(l)}(\infty)$ is the value of the $l$-th component of the vector $\hat{U}_{j 3}(\tau)$ evaluated at $\tau=\infty$, i.e., in the steady-state. $R_{j k}(i z)$ is the $(j, k)$ element of the matrix $R(i z)$ defined as

$$
R(i z)=(i z \hat{I}-M)^{-1}
$$

$\hat{I}$ being the identity matrix with size $8 \times 8, z_{1}=\left(\omega-\omega_{L}\right) / \Gamma_{0}$, and $B_{l}$ is the $l$-th element of column vector $B$.

\section{Appendix B: Appendix B: Resonance fluorescence spectrum in the dressed state} basis

Here we address the problem of computing the RFS in the dressed state picture for the general case of a driving field $\left(\Omega_{3} \neq 0\right)$ out of resonance $(\delta \neq 0)$. This will allow us to obtain an analytical expression for the spectrum. It can be shown that the eigenvalues of the quantum system plus the coherent part of the Hamiltonian are provided by

$$
\begin{aligned}
& \lambda_{+}=\omega_{21}+\frac{\delta}{2}+\sqrt{\left(\frac{\delta}{2}\right)^{2}+\left|\Omega_{3}\right|^{2}}, \\
& \lambda_{-}=\omega_{21}+\frac{\delta}{2}-\sqrt{\left(\frac{\delta}{2}\right)^{2}+\left|\Omega_{3}\right|^{2}},
\end{aligned}
$$

while the corresponding eigenvectors read as

$$
\begin{aligned}
& |+\rangle=c_{2}|2\rangle+c_{3}|3\rangle, \\
& |-\rangle=-c_{3}|2\rangle+c_{2}|3\rangle,
\end{aligned}
$$

where $c_{2}=\operatorname{sign}\left(\omega_{21}-\lambda_{+}\right) \frac{\Omega_{3}}{A}, c_{3}=\frac{\omega_{21}-\lambda_{+}}{A}$, and $A=\sqrt{\left|\Omega_{3}\right|^{2}+\left(\omega_{21}-\lambda_{+}\right)^{2}}$.

The eigenstates in Eq. (B2) define a rotation matrix $T$ that diagonalizes the quantum system and coherent part of the Hamiltonian of Eq. (14) via the matrix product THT $^{-1}$. Thus the density operator in the dressed state basis $\rho^{D}$ will be given by $\rho^{D}=T \rho T^{-1}$, with $\rho$ being the density matrix operator in the bare state basis. Projection of the master equation over the dressed state basis gives rise to complicated couplings between the dressed-state populations and coherences. However, the situation can be simplified in the strong field 
limit where the effective Rabi frequency is much greater than all relaxation rates. In this case, we can ignore the non-secular terms, i.e., coupling between population and coherences.

The Bloch equations in the secular approximation for the coherences are given by

$$
\begin{aligned}
& \frac{d\left\langle\sigma_{+1}(t)\right\rangle}{d t}=-\left(\Gamma_{+1}-i \lambda_{+}\right)\left\langle\sigma_{+1}(t)\right\rangle, \\
& \frac{d\left\langle\sigma_{-1}(t)\right\rangle}{d t}=-\left(\Gamma_{-1}-i \lambda_{-}\right)\left\langle\sigma_{-1}(t)\right\rangle, \\
& \frac{d\left\langle\sigma_{+-}(t)\right\rangle}{d t}=-\left(\Gamma_{+-}+i\left(\lambda_{+}-\lambda_{-}\right)\right)\left\langle\sigma_{+-}(t)\right\rangle,
\end{aligned}
$$

with

$$
\begin{aligned}
\Gamma_{+1} & =\frac{1}{2}\left(\left(\Gamma_{31}+\Gamma_{32}\right)\left|c_{3}\right|^{2}+\left(\gamma_{22}+\Gamma_{21}\right)\left|c_{2}\right|^{2}+\Gamma_{12}\right), \\
\Gamma_{-1} & =\frac{1}{2}\left(\left(\Gamma_{31}+\Gamma_{32}\right)\left|c_{2}\right|^{2}+\left(\gamma_{22}+\Gamma_{21}\right)\left|c_{3}\right|^{2}+\Gamma_{12}\right), \\
\Gamma_{+-} & =\frac{1}{2}\left(\Gamma_{31}+\Gamma_{32}+\Gamma_{21}+\gamma_{22}\right)+\left(\Gamma_{32}-\gamma_{22}\right)\left|c_{2}\right|^{2}\left|c_{3}\right|^{2} .
\end{aligned}
$$

In a similar way we derive the equations of motion for populations which read

$$
\begin{aligned}
& \frac{d\left\langle\sigma_{++}(t)\right\rangle}{d t}=\Gamma_{++}\left\langle\sigma_{++}(t)\right\rangle+\Gamma_{+-}\left\langle\sigma_{--}(t)\right\rangle+\Gamma_{++}^{0}, \\
& \frac{d\left\langle\sigma_{--}(t)\right\rangle}{d t}=\Gamma_{-+}\left\langle\sigma_{++}(t)\right\rangle+\Gamma_{--}\left\langle\sigma_{--}(t)\right\rangle+\Gamma_{--}^{0},
\end{aligned}
$$

with

$$
\begin{aligned}
& \Gamma_{++}=-\left(\Gamma_{31}+\Gamma_{32}\left(1-\left|c_{2}\right|^{2}\right)\right)\left|c_{3}\right|^{2}-\left(\Gamma_{21}+\Gamma_{12}+\gamma_{22}-\gamma_{22}\left|c_{2}\right|^{2}\right)\left|c_{2}\right|^{2}, \\
& \Gamma_{+-}=\left(\Gamma_{32}\left|c_{2}\right|^{2}-\Gamma_{12}+\gamma_{22}\left|c_{3}\right|^{2}\right)\left|c_{2}\right|^{2}, \\
& \Gamma_{++}^{0}=\Gamma_{12}\left|c_{2}\right|^{2}, \\
& \Gamma_{-+}=\left(\Gamma_{32}\left|c_{3}\right|^{2}-\Gamma_{12}+\gamma_{22}\left|c_{2}\right|^{2}\right)\left|c_{3}\right|^{2}, \\
& \Gamma_{--}=-\left(\Gamma_{31}+\Gamma_{32}\left(1-\left|c_{3}\right|^{2}\right)\right)\left|c_{2}\right|^{2}-\left(\Gamma_{21}+\Gamma_{12}+\gamma_{22}-\gamma_{22}\left|c_{3}\right|^{2}\right)\left|c_{3}\right|^{2}, \\
& \Gamma_{--}^{0}=\Gamma_{12}\left|c_{3}\right|^{2} .
\end{aligned}
$$

The RFS is obtained in the dressed state basis by applying the quantum regression theorem to the Eqs. (B33) and (B5). After a lengthy but straightforward calculation, the RFS in this basis is given by

$$
S_{D S P}(\omega)=S_{a}^{D S P}(\omega)+S_{b}^{D S P}(\omega) .
$$


The first term in Eq. (B7) explicitly reads as

$$
S_{a}^{D S P}(\omega) \propto \Re\left\{\left|c_{3}\right|^{2} \frac{\left\langle\sigma_{++}(\infty)\right\rangle}{i\left(\omega-\lambda_{+}\right)+\Gamma_{+1}}+\left|c_{3}\right|^{2} \frac{\left\langle\sigma_{--}(\infty)\right\rangle}{i\left(\omega-\lambda_{-}\right)+\Gamma_{-1}}\right\},
$$

and accounts for the photons arising from transition $|1\rangle \leftrightarrow|3\rangle$, while the remaining term is given by

$$
S_{b}^{D S P}(\omega) \propto \Re\left\{\left|c_{3}\right|^{4} \frac{\left\langle\sigma_{++}(\infty)\right\rangle}{i\left(\omega+\lambda_{+}-\lambda_{-}\right)+\Gamma_{+-}}+\left|c_{2}\right|^{4} \frac{\left\langle\sigma_{--}(\infty)\right\rangle}{i\left(\omega-\lambda_{+}+\lambda_{-}\right)+\Gamma_{+-}}+S_{0}(\omega)\right\}
$$

and accounts for the photons arising from transition $|2\rangle \leftrightarrow|3\rangle$. We have introduced the term $S_{0}(\omega)$ for the central peak of the spectrum: this central peak arises from the contributions of the transitions $|+, N\rangle \leftrightarrow|+, N-1\rangle$, and $|-, N\rangle \leftrightarrow|-, N-1\rangle, N$ being the number of photons in the manifold.

The computation of $S_{0}(\omega)$ requires the solution of the coupled equations (B5) involving the populations. To this end we define the vector $\hat{U}(t)=\left[\left\langle\sigma_{++}(t)\right\rangle,\left\langle\sigma_{--}(t)\right\rangle\right]^{T}$, which satisfies the equation of motion

$$
\frac{d \hat{U}(t)}{d t}=M_{0} \hat{U}(t)+B_{0}
$$

where the coefficients of matrix $M_{0}$ and vector $B_{0}$ can be easily obtained from Eqs. (B5).

It can be shown that term $S_{0}(\omega)$ of the central peak is given by

$$
S_{0}(\omega)=\left|c_{3} c_{2}\right|^{2} \Re\left\{\int_{0}^{\infty} d \tau e^{-i \omega \tau}\left(\left\langle\sigma_{++}(\tau) \sigma_{++}(\infty)\right\rangle+\left\langle\sigma_{--}(\tau) \sigma_{--}(\infty)\right\rangle\right)\right\} .
$$

The two-time correlation functions from equation (B11) can be computed by invoking the quantum regression theorem together with the Eq. (B10). We define the column vector:

$$
\hat{U}^{j}(\tau)=\left[\left\langle\sigma_{++}(\tau) \sigma_{j j}(\infty)\right\rangle,\left\langle\sigma_{--}(\tau) \sigma_{j j}(\infty)\right\rangle\right]^{T} j=+,-.
$$

According to the quantum regression theorem, for $\tau>0$ the vector $\hat{U}^{j}(\tau)$ satisfies the equation

$$
\frac{\partial \hat{U}^{j}(\tau)}{\partial \tau}=M_{0} \cdot \hat{U}^{j}(\tau)+B_{0}\left\langle\sigma_{j j}(\infty)\right\rangle(j=+,-) .
$$

Working with Eq. (B13) in the Laplace space, we obtain the values for the two-time correlation functions appearing in Eq. (B11) which now read

$$
\begin{aligned}
& \int_{0}^{\infty} d \tau e^{-i \omega \tau}\left\langle\sigma_{++}(\tau) \sigma_{--}(\infty)\right\rangle=\sum_{m=1}^{2} R_{1 m}(i z)\left(\hat{U}_{m}^{+}(\tau=0)+\frac{B_{0, m}}{i z}\left\langle\sigma_{++}(\infty)\right\rangle\right) \\
& \int_{0}^{\infty} d \tau e^{-i \omega \tau}\left\langle\sigma_{--}(\tau) \sigma_{--}(\infty)\right\rangle=\sum_{m=1}^{2} R_{2 m}(i z)\left(\hat{U}_{m}^{-}(\tau=0)+\frac{B_{0, m}}{i z}\left\langle\sigma_{--}(\infty)\right\rangle\right),
\end{aligned}
$$


where we have set $z \equiv\left(\omega-\omega_{L}\right) / \Gamma_{0}$, and $\hat{U}_{m}^{j}(\tau=0)$ stands for the $m$-th component of the vector $\hat{U}^{j}(\tau)$ evaluated in the steady-state. Here, $R_{n m}(i z)$ is the $(n, m)$ element of the matrix

$$
R(i z)=\left(i z \hat{I}-M_{0}\right)^{-1}
$$

$\hat{I}$ being the $2 \times 2$ identity matrix, and $B_{0, m}$ is the $m$-th element of the column vector $B_{0}$.

\section{ACKNOWLEDGMENTS}

This work has been supported by Project no. FIS2013-41709-P (MICINN) from Spain. F.C. and M.A. A. thank Complutense University for financial support with grant reference GR3/14-910133.

[1] K. T. Shimizu, W. K. Woo, B. R. Fisher, H. J. Eisler, M. G. Bawendi, Phys. Rev. Lett. 89, 117401 (2002).

[2] W. Zhang, A. O. Govorov, G. W. Bryant, Phys. Rev. Lett. 97, 146804 (2006).

[3] R. D. Artuso, G. W. Bryant, Nano Lett. 8, 2106 (2008).

[4] A. O. Govorov, G. W. Bryant, W. Zhang, T. Skeini, J. Lee, N. A. Kotov, J. M. Slocik, R. R. Naik, Nano Lett. 6, 984 (2006).

[5] V. V. Klimov, M. Ducloy, V. S. Letokhov, Eur. Phys. J. D 20, 133 (2002).

[6] A. O. Govorov, G. W. Bryant, W. Zhang, T. Skeini, J. Lee, N. A. Kotov, J. M. Slocik, R. R. Naik, Nano Lett. 6, 984 (2006).

[7] A. Manjavacas, F. J. García de Abajo, P. Nordlander, Nano Lett. 11, 2318 (2011).

[8] A. Manjavacas, P. Nordlander, F. J. García de Abajo, ACS Nano 2, 1724 (2012).

[9] F. H. L. Koppens, D. E. Chang, F. J. García de Abajo, Nano Lett. 11, 3370 (2011).

[10] T. B. Hoang, G. M. Akselrod, C. Argyropoulos, J. Huang, D. R. Smith, M. H. Mikkelsen, Nature Commun. 6, 7788 (2015).

[11] T. B. Hoang, G. M. Akselrod, M. H. Mikkelsen, Nano Lett. 16, 270 (2016).

[12] D. Ratchford, F. Shafiei, S. Kim, S. K. Gray, X. Li, Nano Lett. 11, 1049 (2011).

[13] A. Ridolfo, O. Di Stefano, N. Fina, R. Saija, S. Savasta, Phys. Rev. Lett. 105, 263601 (2010).

[14] Y. Gu, L. Huang, O. J. F. Martin, Q. Gong, Phys. Rev. B 81, 193103 (2010). 
[15] Y. V. Vladimirova, V. V. Klimov, V. M. Pastukhov, V. N. Zadkov, Phys. Rev. A 85, 053408 (2012).

[16] E. S. Andrianov, A. A. Pukhov, A. P. Vinogradov, A. V. Dorofeenko, A. A. Lisyansky, Jetp Lett. 97452 (2013).

[17] F. Carreño, M. A. Antón, F. Arrieta-Yáñez, Phys Rev. B 88, 195303 (2013).

[18] R.C. Ge, C. Van Vlack, P. Yao, J. F. Young, S. Hughes Phys. Rev. B 87, 205425 (2013).

[19] J. Hakami, L. Wang, M. S. Zubairy, Phys. Rev. A 89, 053835 (2014).

[20] V. Yannopapas, E. Paspalakis, N. V. Vitanov, Phys. Rev. Lett. 103, 063602 (2009).

[21] S. Evangelou, V. Yannopapas, E. Paspalakis, Phys. Rev. A 83, 023819 (2011).

[22] Y. Gu, L. Wang, P. Ren, J.-X. Zhang, T.-C. Zhang, O. J. F. Martin, Q.-H. Gong, Nano Lett. $12,2488(2012)$.

[23] M.-T. Cheng, S.-D. Liu, H.-J. Zhou, Z.-H. Hao, Q.-Q. Wang, Opt. Lett. 32, 2125 (2007).

[24] S. M. Sadeghi, Phys. Rev. B 79, 233309 (2009).

[25] M. A. Antón, F. Carreño, S. Melle, O. G. Calderón, E. Cabrera- Granado, J. Cox, M. R. Singh, Phys. Rev. B 86, 155305 (2012).

[26] E. Paspalakis, S. Evangelou, A. F. Terzis, Phys. Rev. B 87, 235302 (2013).

[27] A. V. Malyshev, V. A. Malyshev, Phys. Rev. B 84, 035314 (2011).

[28] I. Thanopulos, E. Paspalakis, V. Yannopapas, Phys. Rev. B 85, 035111 (2012).

[29] M. R. Singh, Nanotechnology 24, 125701 (2013).

[30] J. D. Cox, M. R. Singh, M. A. Antón, F. Carreño, J. Phys. Condens. Matter. 25, 385302 (2013).

[31] J. D. Cox, M. R. Singh, C. von Bilderling, A. V. Bragas, Adv. Opt. Mater. 1, 460 (2013).

[32] B. S. Nugroho, A. A. Iskandar, V. A. Malyshev, J. Knoester, J. Chem. Phys. 139, 014303 (2013).

[33] E. Paspalakis, S. Evangelou, S. G. Kosionis, A. F. Terzis, J. Appl. Phys. 115083106 (2014).

[34] S. Evangelou, V. Yannopapas, E. Paspalakis, J. Mod. Opt. 61, 1458 (2014).

[35] H. T. Dung, L. Knoll, D. G. Welsch, Phys Rev. A 66, 063810 (2002).

[36] M. O. Scully, M. S. Zubairy, Quantum Optics, 1997 Cambridge University Press.

[37] M. Lax, Phys. Rev. 172, 350 (1968).

[38] P.B. Johnson, R. W. Christy, Phys. Rev. B 6, 4370 (1972).

[39] J. D. Jackson, Classical Electrodynamics, 1999 Wiley, New York. 
[40] V. Yannopapas, N. V. Vitanov, Phys. Rev. B 75, 115124 (2007).

[41] H. J. Kimble, L. Mandel, Phys. Rev. A 13, 2123 (1976).

[42] H. J. Kimble, M. Dagenais, L. Mandel, Phys. Rev. Lett. 39, 691 (1977).

[43] D. Brunner, B. D. Gerardot, P. A. Dalgarno, G. Wüst, K. Karrai, N. G. Stoltz, P. M. Petroff, R. J. Warburton, Science 325, 70 (2009).

[44] A. N. Vamivakas, Y. Zhao, Chao-Yang Lu, M. Atatüre, Nat. Phys. 5, 198 (2009).

[45] H. S. Nguyen, G. Sallen, C. Voisin, Ph. Roussignol, C. Diederichs, G. Cassabois, Appl. Phys. Lett. 99, 261904 (2011).

[46] C. Matthiesen, A. N. Vamivakas, M. Atatüre, Phys. Rev. Lett. 108, 093602 (2012).

[47] K. Konthasinghe, M. Peiris, B. Petrak, Y. Yu, Z. C. Niu, A. Muller, Opt. Lett. 40, 1846 (2015).

[48] G. Fernandez, T. Volz, R. Desbuquois, A. Badolato, A. Imamoglu, Phys. Rev. Lett. 103, $087406(2009)$.

[49] Y. He, Y. -M. He, Y. -J. Wei, X. Jiang, M. -C. Chen, F. -L. Xiong, Y. Zhao, C. Schneider, M. Kamp, S. Höfling, C. -Y. Lu, J. -W. Pan, Phys. Rev. Lett. 111, 237403 (2013).

[50] C. Santori, D. Fattal, K-M. C. Fu, P. E. Barclay, R. G. Beausoleil, New J. Phys. 11, 123009 (2009).

[51] T. M. Sweeney, S. G. Carter, A. S. Bracker, M. Kim, C. S. Kim, L. Yang, P. M. Vora, P. G. Brereton, E. R. Cleveland, D. Gammon, Nat. Phot. 8, 442 (2014).

[52] A. Urbańczyk, G. J. Hamhuis, R. Nötzel, Appl. Phys. Lett. 96, 113101 (2010).

[53] A. G. Curto, G. Volpe, T. H. Taminiau, M. P. Kreuzer, R. Quidant, and N. F. van Hulst, Science 329, 930 (2010).

[54] M. Pfeiffer, K. Lindfors, H. Zhang, B. Fenk, F. Phillipp, P. Atkinson, A. Rastell, O. G. Schmidt, H. Giessen, M. Lippitz, Nano Lett. 14,197 (2014).

[55] S-H. Gong, J-H. Kim, Y-H. Ko, C. Rodriguez, J. Shin, Y-H. Lee, L. S. Dang, X. Zhang, Y-H. Cho, Proc. Nat. Acad. Sci. 112, 5280 (2015).

[56] A. A. Lyamkina, K. Schraml, A. Regler, M. Schalk, A. K. Bakarov, A. I. Toropov, S. P. Moshchenko, M. Kaniber, Monolithically integrated single quantum dots coupled to bowtie nanoantennas, arXiv:1603.07093v1. 